\title{
Meta
}

Journal des traducteurs

Translators' Journal

\section{SELA-SHEFFY, Rakefet et Miriam SHLESINGER (dir.) (2011) : Identity and Status in the Translational Professions. Amsterdam et Philadelphie : John Benjamins Publishing} Company, 282 p.

\section{Valérie Florentin}

Volume 59, numéro 3, décembre 2014

URI : https://id.erudit.org/iderudit/1028664ar

DOI : https://doi.org/10.7202/1028664ar

Aller au sommaire du numéro

Éditeur(s)

Les Presses de l’Université de Montréal

ISSN

0026-0452 (imprimé)

1492-1421 (numérique)

Découvrir la revue

Citer ce compte rendu

Florentin, V. (2014). Compte rendu de [SELA-SHEFFY, Rakefet et Miriam SHLESINGER (dir.) (2011): Identity and Status in the Translational Professions. Amsterdam et Philadelphie : John Benjamins Publishing Company, 282 p.]

Meta, 59(3), 691-692. https://doi.org/10.7202/1028664ar d'utilisation que vous pouvez consulter en ligne. 


\section{DOCUMENTATION}

\section{Comptes rendus}

Sela-Sheffy, Rakefet et Miriam Shlesinger (dir.) (2011): Identity and Status in the Translational Professions. Amsterdam et Philadelphie: John Benjamins Publishing Company, 282 p.

Cet ouvrage collectif de la collection «Benjamins Current Topics» propose quatorze articles déjà publiés dans deux numéros spéciaux de la revue Translation and Interpreting Studies (soit les numéros 4(2) 2009 et 5(1) 2010) et font suite à un colloque tenu à Tel Aviv en 2009. Ces articles sont scindés en deux parties, plutôt floues à la lecture de l'ouvrage, portant sur le statut professionnel des traducteurs et interprètes d'une part et sur leur identité professionnelle d'autre part.

Le domaine d'étude est jeune puisque la place des traducteurs et interprètes est étudiée depuis les années 2000 seulement, en grande partie grâce aux travaux de Gideon Toury sur les normes, et aux travaux de Lawrence Venuti sur l'invisibilité du traducteur. Pour toute personne intéressée par le sujet, l'introduction à elle seule vaut le détour puisqu'elle réussit le tour de force de présenter, en cinq pages à peine, les tenants et aboutissants de la problématique abordée - à savoir définir si la traduction et l'interprétation sont, de nos jours, des occupations ou des professions -, en plus de fournir quatre pages de références bibliographiques qui pourraient s'avérer particulièrement utiles à tout chercheur débutant ou à tout professionnel intéressé par l'un des aspects soulevés. Les paradoxes de la profession sont précisés: le pouvoir culturel potentiel des traducteurs et leur statut obscur et subalterne; leur mépris envers leur propre statut et leur refus d'être des agents du changement, ce qui les pousse à continuer de produire des textes qui respectent les normes établies.

Nous sommes d'avis que la lecture de l'ouvrage aurait été plus aisée avec une table des matières mieux divisée et nous nous proposons de découper en trois parties le vaste sujet abordé ici. La première établira la définition et les enjeux des professions de traducteur et d'interprète; la deuxième, la manière dont les circonstances externes forgent l'identité d'un traducteur; la troisième, le rôle de médiateur culturel endossé par les traducteurs et interprètes.
Première partie: La définition et les enjeux des professions de traducteur et d'interprète

Larticle de David Katan (p. 65- 87) forme, à notre avis, l'introduction parfaite à cette partie puisqu'il dresse le portrait des traducteurs et interprètes du monde entier, grâce à un sondage effectué en 2008 auprès de mille professionnels. Cependant, c'est la mise en relation avec les articles de Robin Setton et Alice Guo Liangliang (p. 89-117), de Franz Pöchhacker (p. 49-63) et de Cornelia Zwischenberger (p. 119-133) qui lui donne toute sa portée, puisque les conclusions de ces quatre articles sont les mêmes:

- les traducteurs souffrent d'un manque de reconnaissance (ils s'estiment aussi valorisés qu'une secrétaire), mais sont heureux; - les interprètes, eux, jouissent d'un statut plus élevé et estiment jouer un rôle très important dans l'acte de communication;

- traducteurs comme interprètes exercent souvent un autre métier;

- de nombreux interprètes sont traducteurs, mais l'inverse n'est pas vrai, ce qui renforce l'idée que tout le monde peut traduire;

- les traducteurs sont généralement moins spécialisés que les interprètes;

- la formation ne cesse jamais, mais les deux professions sont dénuées de structures et il n'y a donc pas de réelle progression;

- les deux professions avouent se livrer à l'autocensure et évitent les gros mots, voire les critiques trop virulentes envers autrui;

- la fidélité est due d'abord au texte ou à l'auteur, puis au public, mais pas vraiment au client.

Pour poursuivre sur le sujet de la professionnalisation des traducteurs, les articles d'Andy Lung Jan Chan (p. 31-48) et d'Esther Monzó (p. 11-30) apportent des pistes de solution. Le premier constate en effet que le niveau de scolarité est le seul moyen de mesurer les aptitudes d'un candidat à un poste de traducteur et qu' il est maintenant possible de suivre une formation universitaire en traduction dans plus de soixante-treize pays, un nombre qui a doublé au cours de la dernière décennie. Ainsi, les recruteurs accordent plus d'importance au diplôme qu'à l'expérience, qui ne suffit plus. La certification, elle, est considérée comme un avantage, sans plus. L'auteur milite donc pour que le milieu professionnel soit consulté afin de revoir les programmes d'étude pour qu'ils correspondent mieux aux attentes du marché. À l’inverse, Monzó 
incite les traducteurs eux-mêmes à se prendre en main et à se regrouper en associations afin de pousser les gouvernements à reconnaître leurs droits en imposant la diplomation et la certification à tous ceux qui voudraient s'improviser traducteurs. Les deux mesures devraient permettre de redorer le blason des traducteurs en leur accordant un statut de professionnels reconnus et, d'autre part, en permettant l'augmentation des tarifs de traduction, qui ne seront plus revus systématiquement à la baisse par la concurrence déloyale de tout amateur qui voudrait obtenir sa part des profits.

\section{Deuxième partie: la manière dont les circons- tances externes forgent l'identité du traducteur}

Cette section regroupe les études de cas réalisées par Reine Meylaerts (p. 135-153), Elena Baibikov (p. 173-188) et Hannah Amit-Kochavi (p. 155172). Le premier article traite d'un traducteur belge du début $\mathrm{du} \mathrm{xx}^{\mathrm{e}}$ siècle, le deuxième d'une traductrice japonaise de la même époque et le dernier des traducteurs littéraires arabe-hébreu vivant en Israël. Ces trois articles décrivent, à l'aide d'exemples concrets, comment le passé des traducteurs étudiés modèle leur rôle et la manière dont ils se perçoivent, mais aussi comment leur vécu influence leurs traductions, tant sur le plan des textes qu'ils choisissent de traduire que sur le plan des choix traductifs.

Troisième partie: Le rôle de médiateur culturel endossé par les interprètes

Cette dernière partie se comprend mieux en conclusion des deux autres, et comporte les études de cas réalisées par Kumiko Torikai (p. 189-207), Ruth Morris (p. 209-229), Claudia V. Angelelli (p. 231-245) et Şebnem Bahadır (p. 263-278). Les différents auteurs s'attachent plus particulièrement à démontrer, là encore par l'exemple, le rôle de médiateurs culturels qu'endossent, souvent sans l'admettre, les interprètes. À ce sujet, Bahadır milite pour que soit révisé le mode de formation des interprètes afin qu'ils soient prêts à endosser pleinement leur rôle. En effet, Bahadır fait valoir que la professionnalisation des interprètes nuit à leur mandat puisque ces derniers respectent des normes qui renforcent l'écart entre la langue/ culture dominante et la langue/culture dominée, empêchant ainsi l'interprète de demeurer neutre, l'obligeant à prendre parti.

Pour terminer notre tour d'horizon, mentionnons que l'article de Nadja Grbić (p. 247-261) a comme seul avantage de proposer une définition de la classification («boundary work») et de son utilité pour le domaine. Le reste de l'article est malheureusement composé de questions qui resteront sans réponse.
En conclusion, si la première partie dresse un portrait assez sombre du métier de traducteur (à défaut de celui d'interprète), les deux autres mettent au jour des facettes intéressantes de ces métiers, souvent mentionnées mais peu étudiées, notamment l'influence de la vie privée du traducteur sur ses traductions et le rôle de médiateur culturel endossé par les interprètes. Par contre, à la question posée en introduction quant à savoir si traducteur et interprète sont des occupations ou de réelles professions, de nombreux facteurs (notamment l'absence de structure professionnelle, l'accès au marché du travail par les amateurs et le fait que traducteurs et interprètes ont presque tous un autre métier) indiquent que, malheureusement, il s'agit d'occupation et ce, même si les interprètes semblent mieux lotis. Il serait peut-être temps, comme l'indiquent certains auteurs, que les professionnels concernés s'unissent et fassent reconnaître leurs droits.

VALÉRIE FLORENTIN Université de Montréal, Canada

De Jongh, Elena M. (2012): From the classroom to the courtroom: A guide to interpreting in the U.S. justice system. Amsterdam: John Benjamins Publishing Company, 215 p.

Dans un système judiciaire contradictoire, un procès se déroule comme une joute opposant la version de l'accusation à la version de la défense. Ce combat singulier, cependant, s'appuie sur l'apport de nombreux acteurs du processus judiciaire, dont le juge, les témoins et l'accusé. Lorsque l'accusé ou les témoins ne maîtrisent pas la langue dans laquelle se déroule par ailleurs le procès, il faut, pour que celui-ci ait lieu de manière équitable, faire entrer en piste un acteur essentiel: l'interprète judiciaire. C'est à ce personnage clé que Elena M. de Jongh, qui enseigne l'interprétation au Département de langues modernes de la Florida International University et agit depuis 1985 comme interprète judiciaire agréée, consacre son ouvrage.

Comme le titre l'indique, cet ouvrage est conçu comme un guide pratique d'exercice de l'interprétation dans le cadre du système judiciaire aux États-Unis. Elena M. de Jongh nous avait déjà offert en 1992 un ouvrage intitulé An introduction to court interpreting: Theory and practice. L'intérêt du premier opus était lié entre autres au faible nombre de livres consacrés à l'interprétation judiciaire et au nombre plus faible encore d'ouvrages didactiques, ancrés dans la pratique. Dans ce deuxième opus, Elena $\mathrm{M}$. de Jongh persiste et propose au futur interprète une incursion très réaliste dans la pratique de la profession. 